\title{
Prevalência, fatores associados e desfechos reprodutivos relacionados ao ganho de peso gestacional excessivo
}

\author{
Prevalence, associated factors and reproductive outcomes related to excessive gestational weight gain
}

Prevalencia, factores asociados y resultados reproductivos relacionados con el aumento excesivo de peso durante la gestación

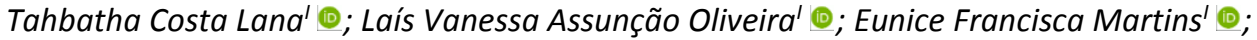 \\ Nágela Cristine Pinheiro Santos ${ }^{\circledR}$; ; Fernanda Penido Matozinhos ${ }^{\prime} \odot$; Mariana Santos Felisbino-Mendes ${ }^{\prime} \odot$
}

'Universidade Federal de Minas Gerais, Belo Horizonte, MG, Brasil

\begin{abstract}
RESUMO
Objetivo: avaliar o estado nutricional pré-gestacional, o ganho de peso durante a gestação, e investigar fatores associados ao ganho de peso excessivo (GPE) entre mulheres. Método: estudo com dados secundários de 747 puérperas da pesquisa "Nascer em Belo Horizonte". Calculou-se prevalência, razão de prevalência e intervalos de $95 \%$ de confiança. Utilizou-se teste quiquadrado de Pearson para avaliar diferenças das prevalências. Resultados: de acordo com os dados, $31 \%$ tinham excesso de peso pré-gestacional e $21 \%$ apresentaram GPE na gestação. Mulheres com baixa escolaridade (26,9\%), multíparas (32,0\%), que consumiam álcool $(29,5 \%)$, com pré-natal no serviço público $(25,4 \%)$, poucas consultas $(26,5 \%)$, e gestação de risco $(33,9 \%)$ apresentaram maior GPE. Tiveram maior chance de GPE mulheres com baixa escolaridade, pré-natal em serviço público e gestação de risco. Cesariana $(52,6 \%)$ e macrossomia $(6,6 \%)$ foram mais prevalentes entre aquelas com GPE. Conclusão: observou-se excesso de peso pré-gestacional, ganho ponderal excessivo na gravidez, principalmente em gestantes com maior vulnerabilidade social, resultando em desfechos reprodutivos desfavoráveis.
\end{abstract}

Descritores: Ganho de Peso; Gestantes; Cuidado Pré-Natal; Enfermagem Obstétrica.

\section{ABSTRACT}

Objective: to evaluate pre-gestational nutritional status and weight gain during pregnancy, and to investigate factors associated with excessive weight gain (EWG) among women. Method: this study used secondary data on 747 women following childbirth in the "Born in Belo Horizonte" study. Prevalence, prevalence ratio and 95\% confidence intervals were calculated. Differences in prevalence were assessed by Pearson chi-square test. Results: according to the data, 31\% were overweight before pregnancy and 21\% had EWG during pregnancy. EWG was higher among women with little schooling (26.9\%), multiparous women (32.0\%), those who consumed alcohol (29.5\%), had antenatal care in the public service (25.4\%), attended few appointments $(26.5 \%)$ or had high-risk pregnancies (33.9\%). Women with little schooling, antenatal care in public service, and high-risk pregnancies were more likely to have EWG. Caesarian delivery (52.6\%) and macrosomia (6.6\%) were more prevalent among those with EWG. Conclusion: Excess weight before pregnancy and excessive weight gain during pregnancy were observed, especially among more socially vulnerable women, resulting in unfavorable reproductive outcomes.

Descriptors: Weight Gain; Pregnant Women; Prenatal Care; Obstetric Nursing.

\section{RESUMEN}

Objetivo: evaluar el estado nutricional pregestacional y el aumento de peso durante el embarazo, e investigar los factores asociados con el aumento de peso excesivo (EWG) entre las mujeres. Método: este estudio utilizó datos secundarios de 747 mujeres después del parto en el estudio "Nacidos en Belo Horizonte". Se calcularon la prevalencia, la razón de prevalencia y los intervalos de confianza del 95\%. Las diferencias en la prevalencia se evaluaron mediante la prueba de chi-cuadrado de Pearson. Resultados: según los datos, el $31 \%$ tenía sobrepeso antes del embarazo y el $21 \%$ tenía EWG durante el embarazo. El GTE fue mayor entre las mujeres con poca escolaridad $(26,9 \%)$, las multíparas $(32,0 \%)$, las que consumían alcohol $(29,5 \%)$, tenían atención prenatal en el servicio público $(25,4 \%)$, asistían a pocas citas $(26,5 \%)$ o tenían embarazos de alto riesgo $(33,9 \%)$. Las mujeres con poca escolaridad, atención prenatal en el servicio público y embarazos de alto riesgo tenían más probabilidades de tener EWG. El parto por cesárea $(52,6 \%$ ) y la macrosomía $(6,6 \%)$ fueron más frecuentes entre las personas con EWG. Conclusión: Se observó exceso de peso antes del embarazo y aumento de peso excesivo durante el embarazo, especialmente entre las mujeres más vulnerables socialmente, lo que resultó en resultados reproductivos desfavorables.

Descriptores: Aumento de Peso; Mujeres Embarazadas; Atención Prenatal; Enfermería Obstétrica.

\section{INTRODUÇÃo}

As mudanças na gestação exigem aportes energéticos maiores ${ }^{1}$, não devendo ser inferior a 1800 quilocalorias (kcal) ao dia ${ }^{2}$. Nessa fase, a mulher deve diversificar os alimentos, garantir uma alimentação rica em proteínas (10-35\%), lipídios (20-35\%), carboidratos (46-65\%), vitaminas e minerais², e suprir essas necessidades energéticas e nutricionais para o desenvolvimento e crescimento fetal adequado ${ }^{1,2}$. 
O ganho ponderal fisiológico ocorre em função do desenvolvimento do concepto e anexos, como placenta, membranas e líquidos ${ }^{3}$, devendo atender limites recomendados que dependem do peso pré-gestacional. 0 ganho total durante a gestação e o ganho semanal médio no 2 ㅇ e no 3 trimestres é de 12,5 a 18 quilogramas $/ \mathrm{kg}(0,51 \mathrm{~kg} / \mathrm{semanas})$ para mulheres com baixo peso, 11,5 a $16 \mathrm{~kg}(0,42 \mathrm{~kg} /$ semana) para eutróficas, 7 a 11,5 kg (0,28kg/semana) e 5 a $9 \mathrm{~kg}$ $\left(0,22 \mathrm{~kg} /\right.$ semana), para sobrepeso e obesas, respectivamente $\mathrm{e}^{4,5}$.

Esses limites tâm sido questionados uma vez que considera adequado uma mulher saudável ganhar até $16 \mathrm{~kg}$, mais do que o dobro do que já foi demonstrado ser suficiente $(7 \mathrm{Kg})^{3,6}$. Além disso, o ganho de peso excessivo (GPE) pode trazer malefícios para o desenvolvimento fetal, e desfechos reprodutivos desfavoráveis ${ }^{1,6}$, como macrossomia ${ }^{7}$, diabetes gestacional ${ }^{7,8}$, eclampsia ${ }^{9}$ e morte materna ${ }^{10}$.

O cuidado pré-natal objetiva acompanhar a gestação, garantindo desenvolvimento fetal adequado, sendo uma oportunidade para aconselhamento da alimentação saudável ${ }^{11}$. Contudo, ainda se observa problemas relacionados à qualidade da assistência, como ausência de avaliação e orientações sobre ganho de peso, ou não avaliação crítica dos riscos do GPE ${ }^{11}$

A crescente e elevada prevalência de sobrepeso e obesidade na população brasileira na última década ${ }^{12}$, seguindo tendências globais, e incluindo mulheres jovens em idade reprodutiva, antes mesmo de engravidar ${ }^{13}$, o que aumenta as chances de desfechos obstétricos desfavoráveis, reforça essa preocupação. Considerando esse aumento da obesidade e a importância de se identificar e orientar quanto ao ganho de peso gestacional adequado, além dos riscos para mãe/feto/recém-nascido, o objetivo deste estudo foi avaliar o estado nutricional pré-gestacional, o ganho de peso durante a gestação, e investigar os fatores associados ao GPE entre as mulheres.

\section{MÉTODO}

Trata-se de estudo com dados da pesquisa "Nascer em Belo Horizonte: Inquérito sobre parto e nascimento", uma coorte retrospectiva de base hospitalar, conduzida em 2011-2012. Foram entrevistadas 1.088 puérperas, uma amostra significativa para o município. Esse estudo seguiu a mesma metodologia da pesquisa nacional "Nascer no Brasil: inquérito sobre o parto e nascimento" ${ }^{14}$, porém diferentemente das demais capitais brasileiras, foram incluídas 11 das 14 maternidades do município.

Procedeu-se com a análise dos dados no segundo semestre de 2019. Foram excluídas as puérperas com registros incompletos para as principais variáveis de interesse. Mulheres sem registros para as duas variáveis que compõem o ganho de peso $(n=115)$, sem registros de peso pré-gestacional ou altura $(n=177)$ e portadoras de doenças prévias (doenças cardíacas, hipertensão arterial com tratamento continuado, anemia grave, lúpus eritematoso sistêmico, hipertireoidismo, diabetes não gestacional, doença renal crônica, epilepsia e acidente vascular encefálico), segundo registros hospitalares $(n=49)$. Ao final, participaram 747 mulheres. Realizou-se análise de sensibilidade e não houve diferenças da ocorrência dos desfechos entre os grupos.

Determinou-se o ganho de peso gestacional a partir das seguintes perguntas: "Qual era seu peso antes de ficar grávida?" e "Qual foi o seu peso na última consulta de pré-natal?", ambas registradas em Kg. A subtração do peso final pelo peso anterior à gravidez resultou no ganho de peso total durante a gestação que, posteriormente, foi dividido pela idade gestacional corrigida, obtendo-se, assim, o ganho de peso semanal.

A idade gestacional (IG) foi calculada com o uso de um algoritmo que ordenou os métodos de estimação da IG ao nascimento, seguindo a ordem decrescente de preferência dos métodos: 1- ultrassonografia em qualquer IG registrada no cartão de pré-natal, 2- IG baseada na ultrassonografia registrada no prontuário da mulher, 3- IG referida pela mulher na entrevista, 4- IG baseada na data da última menstruação (DUM) e registrada no prontuário da mulher, 5- DUM registrada no prontuário da puérpera e 6- DUM registrada na entrevista da puérpera.

O Índice de Massa Corporal (IMC) pré-gestacional foi utilizado para detectar casos de obesidade ou desnutrição antes da gestação, ou até 13 semanas de gestação, e foi calculado a partir do peso e altura anteriores à gravidez, coletados na entrevista. Foi utilizada a fórmula "IMC=Peso/(altura)" e os resultados foram assim classificados: eutróficas $\left(\geq 18,5-24,9 \mathrm{~kg} / \mathrm{m}^{2}\right)$, desnutridas $\left(<18,5 \mathrm{~kg} / \mathrm{m}^{2}\right)$, sobrepeso $\left(\geq 25-29,9 \mathrm{~kg} / \mathrm{m}^{2}\right)$ e obesas $\left(\geq 30 \mathrm{~kg} / \mathrm{m}^{2}\right)$, em consonância com as recomendações nacionais e internacionais ${ }^{4}$.

O GPE foi classificado sim (1) e não (0), baseado nos valores máximos de ganho de peso semanal obtido de acordo com a categoria do IMC pré-gestacional. A metodologia utilizada atende as recomendações internacionais mais recentes ${ }^{4}$ adotadas pelo Ministério da Saúde ${ }^{5}$.

Calculou-se a prevalência para cada categoria do IMC pré-gestacional. Para cada categoria foi calculada a média e desvio padrão (DP) do ganho de peso total e do ganho de peso semanal durante a gestação. Em seguida, calculou-se a 
prevalência, razão de prevalências (RP) e respectivos intervalos de 95\% de confiança (IC95\%) do GPE, segundo fatores sociodemográficos, faixa etária (14-19, 20-29, 30-39 e 40-55 anos), escolaridade (0-8, 9-11, 12 ou mais anos de estudo), cor de pele (branca, preta/parda/morena/mulata, amarelo/oriental/indígena), estado civil (com ou sem companheiro), ocupação (trabalha ou não), paridade (primípara, secundípara e multípara), número de moradores no domicílio $(1-4, \geq 5)$, consumo de álcool e tabaco durante a gestação (sim, não), e assistenciais local de atendimento do pré-natal (particular, público ou os dois), número de consultas pré-natal $(\geq 6,<6)$ e classificação de risco (gestante de risco: sim ou não). A RP foi estimada por meio da Regressão de Poisson ${ }^{15}$. Foi utilizado o teste de qui-quadrado de Pearson para avaliar as diferenças das prevalências e valor- $p \leq 0,05$.

Ao final, estimou-se a prevalência, razão de chances (OR) e intervalos de $95 \%$ de confiança dos desfechos reprodutivos imediatos, na presença de GPE, por meio da regressão logística - tendo em vista desfechos reprodutivos raros, à exceção da cesariana. Considerou-se como desfechos reprodutivos a via de nascimento (vaginal e cesariana), prematuridade (sim, não), baixo peso ao nascer (sim, não) e macrossomia (sim, não).

O estudo "Nascer em Belo Horizonte: Inquérito sobre parto e nascimento" foi aprovado pelo Comitê de Ética em Pesquisa da Universidade Federal de Minas Gerais (UFMG) sob o parecer no 2.804.828, autorizado pelos dirigentes das maternidades, e consentidas pelas mulheres ou seus representantes legais, nos casos de adolescentes, pela assinatura do Termo de Consentimento Livre e Esclarecido.

\section{RESULTADOS}

Observou-se que $31 \%$ das mulheres tinham excesso de peso antes de engravidar. A média ( $\pm D P$ ) de ganho de peso total durante a gestação variou de $8,6( \pm 1,07) \mathrm{Kg}$ para as mulheres com obesidade a $13,0( \pm 0,25) \mathrm{Kg}$ para as mulheres eutróficas (Tabela 1).

TABELA 1: IMC pré-gestacional e ganho de peso médio durante a gestação, total e semanal. Belo Horizonte, MG, Brasil, 2012

\begin{tabular}{|c|c|c|c|c|}
\hline \multirow[b]{2}{*}{ IMC pré-gestacional } & \multirow{2}{*}{$\mathbf{n}$} & \multirow{2}{*}{$\%$} & \multicolumn{2}{|c|}{ Ganho de peso na gestação } \\
\hline & & & Média total (DP)- Kg & Média semanal *(DP) -Kg \\
\hline Eutrofia & 470 & 62,9 & $13,0(0,25)$ & $0,337(0,06)$ \\
\hline Desnutrição & 45 & 6,0 & $12,8(0,70)$ & $0,332(0,18)$ \\
\hline Sobrepeso & 157 & 21,0 & $11,6(0,52)$ & $0,301(0,13)$ \\
\hline Obesidade & 75 & 10,0 & $8,6(1,07)$ & $0,222(0,02)$ \\
\hline Total & 747 & 100,0 & & \\
\hline
\end{tabular}

Ganho de peso semanal, corrigido pela IG padrão ouro. IMC - Índice de Massa Corporal; BH - Belo Horizonte; DP - Desvio-padrão; Kg- Quilogramas.

Aproximadamente $21 \%$ das mulheres apresentaram GPE na gestação. Além disso, observou-se associação dos fatores sociodemográficos e assistenciais com o ganho excessivo (Tabela 2). A maior prevalência de GPE foi observada entre mulheres de 20-39 anos (21,4\% entre 20-29 anos e 22,4\% entre 30-39 anos), com baixa escolaridade (26,9\%), preta/parda/morena/mulata $(22,1 \%)$, sem companheiro $(23,3 \%)$, que moravam em casas com mais de 5 pessoas (27,3\%), multíparas (32,0\%), que consumiam álcool (29,5\%) e tabaco (29,8\%), que fizeram acompanhamento pré-natal no serviço público $(25,4 \%)$, com poucas consultas de pré-natal $(26,5 \%)$ e gestação de risco $(33,9 \%)$.

Observou-se que mulheres com baixa escolaridade (0-8 anos de estudo) apresentaram maior razão de prevalência (RP) de GPE em relação às mulheres com alta escolaridade (12 ou mais) (RP=1,76; IC95\% 1,08-2,86). Também se observou associação do acompanhamento do pré-natal no serviço público (RP=1,59; IC95\% 1,13-2,23) e gestação de alto risco ( $R P=1,84$; IC95\% 1,25-2,71) com o GPE.

Ao se considerar o GPE como risco para desfechos reprodutivos, observou-se maior prevalência de cesariana $(52,6 \%)$ e macrossomia $(6,6 \%)$ entre as mulheres que ganharam peso acima do recomendado, mas sem significância estatística (Tabela 3). 
TABELA 2: Prevalência e Razão de Prevalência de ganho de peso semanal excessivo durante a gestação segundo fatores sociodemográficos e assistenciais. Belo Horizonte, MG, Brasil, 2012.

\begin{tabular}{|c|c|c|c|c|c|}
\hline \multirow{2}{*}{ Fatores sociodemográficos } & \multirow{2}{*}{$\begin{array}{c}\text { População total } \\
\text { (n=747) } \\
n(\%)\end{array}$} & \multicolumn{4}{|c|}{ Ganho de peso semanal excessivo $(n=156)$} \\
\hline & & $\mathbf{n}$ & $\%$ & Valor-p* & RP (IC 95\%) \\
\hline Idade (anos) & & & & 0,545 & \\
\hline $14-19$ & $77(10,3)$ & 12 & 15,9 & & Ref. \\
\hline $30-39$ & $293(39,2)$ & 65 & 22,4 & & $1,42(0,77-2,63)$ \\
\hline $40-55$ & $26(3,5)$ & 04 & 15,4 & & $0,99(0,32-3,06)$ \\
\hline Escolaridade (anos de estudo) & & & & 0,035 & \\
\hline 12 ou mais & $170(22,8)$ & 26 & 15,3 & & Ref. \\
\hline Branca & $214(28,6)$ & 37 & 17,3 & & Ref. \\
\hline Preta/parda/morena/mulata & $507(67,9)$ & 112 & 22,1 & & $1,28(0,88-1,85)$ \\
\hline Amarelo/oriental/indígena & $26(3,5)$ & 07 & 26,9 & & $1,56(0,69-3,49)$ \\
\hline Estado civil & & & & 0,353 & \\
\hline Com companheiro & $567(75,9)$ & 114 & 20,1 & & Ref. \\
\hline Sem companheiro & $180(24,1)$ & 42 & 23,3 & & $1,16(0,81-1,65)$ \\
\hline Trabalho (remunerado) & & & & 0,841 & \\
\hline Paridade & & & & 0,016 & \\
\hline Primípara & $53(13,7)$ & 15 & 28,3 & & Ref. \\
\hline Secundípara & $210(54,1)$ & 39 & 18,6 & & $0,66(0,36-1,19)$ \\
\hline Multípara & $125(32,2)$ & 40 & 32,0 & & $1,13(0,62-2,05)$ \\
\hline Consumo de álcool & & & & 0,029 & \\
\hline Não & $650(87,3)$ & 128 & 19,7 & & Ref. \\
\hline Sim & $95(12,7)$ & 28 & 29,5 & & $1,50(0,99-2,25)$ \\
\hline Fumo durante a gravidez & & & & 0,084 & \\
\hline Não & $690(92,4)$ & 139 & 20,1 & & Ref. \\
\hline Sim & $57(7,6)$ & 17 & 29,8 & & $1,44(0,86-2,41)$ \\
\hline \multicolumn{6}{|l|}{ Fatores Assistenciais } \\
\hline Local do acompanhamento pré-natal & & & & 0,002 & \\
\hline Particular & $313(41,9)$ & 50 & 16,0 & & Ref. \\
\hline Público & $390(52,2)$ & 99 & 25,4 & & $1,59(1,13-2,23)$ \\
\hline Ambos & $44(5,9)$ & 07 & 15,9 & & $1,00(0,45-2,20)$ \\
\hline
\end{tabular}

*Valor-p para o teste Qui-quadrado de Pearson / valores em negrito - significativos ( $<0,05)$; RP - Razão de prevalências. 
Tabela 3: Prevalência e Razão de Chances de desfechos reprodutivos na presença de ganho de peso semanal excessivo durante a gestação. Belo Horizonte, MG, Brasil, 2012.

\begin{tabular}{|c|c|c|c|c|}
\hline \multirow{2}{*}{ Desfechos Reprodutivos } & \multicolumn{4}{|c|}{ Ganho de peso semanal excessivo } \\
\hline & $\mathbf{N}$ & $\%$ & Valor $\mathrm{p}^{*}$ & OR (IC 95\%) \\
\hline \multicolumn{5}{|l|}{ Via de nascimento (cesariana) } \\
\hline Ganho de peso excessivo & & & 0,067 & \\
\hline Não & 262 & 44,3 & & Ref. \\
\hline Sim & 82 & 52,6 & & $1,39(0,98-1,98)$ \\
\hline \multicolumn{5}{|l|}{ Prematuridade } \\
\hline Ganho de peso excessivo & & & 0,362 & \\
\hline Não & 64 & 10,8 & & Ref. \\
\hline Sim & 13 & 8,3 & & $0,75(0,40-1,40)$ \\
\hline Baixo peso ao nascer & & & 0,727 & \\
\hline \multicolumn{5}{|l|}{ Ganho de peso excessivo } \\
\hline Não & 52 & 10,6 & & Ref. \\
\hline Sim & 13 & 9,6 & & $0,89(0,47-1,69)$ \\
\hline Macrossomia & & & 0,170 & \\
\hline \multicolumn{5}{|l|}{ Ganho de peso excessivo } \\
\hline Não & 19 & 3,9 & & Ref. \\
\hline Sim & 09 & 6,6 & & $1,76(0,78-3,99)$ \\
\hline
\end{tabular}

\section{DISCUSSÃo}

Os resultados evidenciaram ocorrência de excesso de peso no início da gestação em 1 a cada 3 mulheres e GPE em 1 a cada 5 gestantes, principalmente entre aquelas com maior vulnerabilidade. As desigualdades socioeconômicas, como baixa escolaridade, paridade, hábitos de vida não saudáveis como consumo de álcool e tabaco, foram mais prevalentes entre mulheres que tiveram GPE na gestação. Identificou-se, também, um GPE entre mulheres com gestação de alto risco, multíparas, que realizaram pré-natal em serviços públicos e tiveram um número de consultas inferior a seis. Ademais, o ganho médio total e o ganho médio semanal se mantiveram dentro das faixas preconizadas pelo Ministério da Saúde.

Destaca-se o sobrepeso e obesidade entre as mulheres em idade fértil antes de engravidar, evento muito prevalente neste estudo, corroborando achados recentes com prevalência cada vez maior desses agravos entre mulheres brasileiras em idade reprodutiva ${ }^{13}$, acompanhando tendências globais, como nos Estados Unidos ${ }^{16}$ e achados para a população brasileira, em todas as unidades federativas ${ }^{12}$. Sabe-se que essa condição está relacionada às repercussões negativas a saúde materna e fetal ${ }^{8,10,17}$, indicando que o planejamento reprodutivo poderia minimizar esses desfechos ${ }^{18}$.

O fato de um quinto das mulheres estudadas terem apresentado GPE reforça a necessidade de maior atenção ao aconselhamento do ganho de peso durante o pré-natal. O GPE, associado à alimentação inadequada, também pode trazer impactos negativos para o crescimento e desenvolvimento do feto ${ }^{16}$, e para desenvolvimento de comorbidades em gestações futuras ${ }^{19}$.

Culturalmente, acredita-se que a gestante deve aumentar a ingesta calórica ${ }^{11}$, além de serem desencorajadas a praticar atividade física ${ }^{20}$. O aumento da ingesta calórica é uma realidade, entretanto, é importante diversificar os alimentos ingeridos, garantindo uma alimentação balanceada e de qualidade ${ }^{2}$. Além disso, as atividades físicas de leve a moderada intensidade contribuem para a saúde da mulher antes, durante e após a gestação e para o desenvolvimento fetal $^{20}$. Ressalta-se a necessidade de desenvolver e intensificar estratégias de cuidado pré-concepcional, pré-natal e puerperal, de forma a estimular as mulheres a adotarem hábitos de vida saudáveis, como alimentação e prática de atividade física regular ${ }^{3,19,21}$.

Gestantes que consumiram álcool e tabaco durante a gestação apresentaram maior GPE, revelando um padrão de acúmulo de comportamentos negativos em saúde, previamente demonstrado ${ }^{13}$. A ingestão moderada a frequente de bebidas alcoólicas também está relacionada ao aumento de gordura corporal e abdominal, devido à quantidade de calorias que cada grama de álcool metabolizado fornece ao organismo ${ }^{22}$. São muitos os riscos do álcool e tabaco para o concepto, como anomalias físicas, risco de abortamento e crescimento intrauterino restrito ${ }^{23}$. Tal fato reforça a necessidade de abordar hábitos de vida mais saudáveis destacando os riscos do uso de qualquer quantidade de bebidas alcoólicas durante a gestação, bem como o uso do tabaco 23 .

Outro resultado importante foi a associação entre fatores que indicam maior vulnerabilidade social com o GPE, revelando presença de iniquidades em saúde, pois mulheres com baixa escolaridade, o que pode refletir pior situação 
socioeconômica, tiveram maior chance do desfecho. Gestantes que possuem menor poder aquisitivo consomem mais frequentemente alimentos com alto teor calórico e baixo valor nutricional, pois possuem menor custo ${ }^{1,19}$. Soma-se a isso, outras vulnerabilidades, como viver em domicílios com mais de cinco moradores, cor da pele preta/parda/morena/mulata e multiparidade. Estas mulheres necessitam de estratégias incorporadas ao cuidado prénatal capazes de reduzir os impactos da sua condição de vida na sua própria saúde e do concepto ${ }^{18}$. Ainda em relação à multiparidade, destaca-se que a cada gestação pode ocorrer aumento de, aproximadamente, um quilograma em relação ao peso anterior e maior chance de retenção de peso pós-parto ${ }^{19}$.

Assim, parece haver um perfil de mulheres mais vulneráveis socialmente que são susceptíveis ao GPE, por acumularem fatores de risco. Apesar de não terem sido encontradas associações entre todos os fatores socioeconômicos e sociodemográficos com o GPE, observa-se que existe diferenças na prevalência e gradiente, os quais poderiam indicar iniquidade no ganho de peso. Esse achado aponta à equipe multidisciplinar quais grupos se beneficiariam mais de ações de educação em saúde.

Observou-se que mulheres com gestação de alto risco tiveram maior ganho ponderal, mesmo realizando pré-natal de alto risco (PNAR), atribuindo ainda mais gravidade a sua saúde. O ganho de peso por essas mulheres poderia estar relacionado às suas comorbidades que contribuem para o acúmulo de peso ${ }^{21}$ ou impedem que pratiquem atividade física. Por isso deve-se garantir maior qualidade do atendimento pré-natal, com diagnóstico precoce da doença de base ou adquirida durante a gestação, e encaminhamento adequado ao PNAR, quando indicado ${ }^{24}$.

O maior ganho de peso em gestantes acompanhadas no serviço público e com menor número de consultas prénatal, pode apontar maior vulnerabilidade dessas mulheres, conforme já discutido, reforçando a importância da qualidade do cuidado pré-natal. Houve, no Brasil, uma expansão importante da cobertura e consequente aumento do acesso a essa assistência ${ }^{25}$. Todavia, a qualidade da assistência também precisa avançar, pois se observa uma atenção com base em tecnologias duras, como exames laboratoriais e ultrassonografias ${ }^{26}$, negligenciando outros cuidados menos densos tecnologicamente, como aferição de pressão, cálculo de IMC e preenchimento de gráficos na caderneta $^{26,27}$. Outra questão seria adoção das tecnologias leves, como escuta atenta, para dialogar com as demais tecnologias e responder as necessidades singulares do cuidado ${ }^{28}$.

Salienta-se que a(o) enfermeira(o) está qualificado para realizar essa assistência, atuando com estratégias de promoção à saúde e prevenção das doenças. Nesse sentido, destaca-se que o aspecto nutricional, enquanto necessidade fisiológica está incluída nos diagnósticos e intervenções de enfermagem e as intervenções de enfermagem incluem cuidados, ensino e monitorização das ações desenvolvidas e ou pactuadas com a mulher ${ }^{29}$.

Os resultados deste estudo apontam quais seriam algumas demandas de cuidados na prática. O ideal seria iniciar com cuidados pré-concepcionais, por meio de orientações sobre a importância de perder e controlar o peso antes da gravidez e incentivo a prática de atividade física. No pré-natal, cabe aos profissionais orientar a gestante, de forma que os mitos relacionados à alimentação e à prática de atividade física sejam desfeitos, aconselhar a mulher e familiares sobre os riscos de complicações pelo GPE, além de acompanhar e aconselhar o ganho de peso adequado e registrar corretamente os achados na caderneta e nos relatórios de saúde.

Esse estudo também mostrou que o GPE se relaciona a ocorrência de desfechos reprodutivos desfavoráveis, como cesarianas e macrossomia. Mesmo esse resultado não apresentando significância estatística, estudos prévios demonstraram essa relação com o aumento da cultura de cesarianas no país ${ }^{30}$, contribuindo para prematuridade, comorbidades materno-infantis e aumento da mortalidade materna e neonatal ${ }^{31}$.

\section{Limitações do estudo}

Uma limitação foi a impossibilidade de estudar mulheres sem registros de peso ou altura, pois estas apresentaram maior proporção de características de maior vulnerabilidade social, tais como baixa escolaridade, cor da pele preta e parda, adolescentes, multíparas, mulheres que bebem e fumam, que não trabalham, sem companheiro e que residiam com mais de 5 pessoas, conforme análise das perdas. Esse viés poderia levar a uma subestimação das relações entre o GPE em mulheres com maior situação de vulnerabilidade, e ainda aponta para iniquidades na atenção pré-natal, pois os piores registros estão entre aquelas que mais se beneficiariam da vigilância em relação ao ganho de peso, conforme já demostrado ${ }^{13}$.

Por outro lado, pode ser visto como aspecto positivo do estudo o fato de ter como base os dados proveniente de importante estudo relacionado à assistência pré-natal, ao parto e nascimento. Tais dados, ainda que referentes ao período de 2011-2012, são dados de base para nortear políticas de saúde no campo obstétrico. 


\section{CONCLUSÃO}

Este estudo evidenciou ocorrência de excesso de peso no início da gestação, e ganho ponderal excessivo durante a gravidez, principalmente para as gestantes em condições de maior vulnerabilidade demonstrando inequidades e resultando em desfechos reprodutivos desfavoráveis. Tal fato reforça a necessidade de maior qualificação do cuidado antes e durante o período gravídico-puerperal e sua associação com as doenças e agravos crônicos não transmissíveis, com maior ênfase aos grupos mais vulneráveis.

Considerando o crescente aumento da obesidade na população brasileira na última década, e que o GPE aumenta as chances de desfechos obstétricos desfavoráveis, reforça a necessidade de incorporar ao cuidado pré-natal essa abordagem, pois trata-se de oportunidades múltiplas a cada consulta para o aconselhamento da mudança de hábitos de vida que beneficiarão a saúde do binômio.

\section{REFERÊNCIAS}

1. Teixeira CSS, Cabral ACV. Nutritional Status of Pregnant Women under Monitoringin Pre Distinct Prenatal Services: The Metropolitan Areaand the Rural Environment. Rev. Bras. Ginecol. Obstet. [Internet]. 2016 [cited 2019 Nov 15]; 38(1):27-34. DOI: https://doi.org/10.1055/s-0035-1570111.

2. Castro P da S, Castro MBT de, Kac G. Adherence to dietary recommendations by the Institute of Medicine and the effect on body weight during pregnancy. Cad. Saúde Pública [Internet]. 2013 [cited 2019 Nov 15]; 29(7):1311-21. DOI: https://doi.org/10.1590/S0102-311X2013000700006.

3. Rogozińska E, Zamora J, Marlin N, Betrán AP, Astrup A, Bogaerts A, et al. Gestational weight gain outside the Institute of Medicine recommendations and adverse pregnancy outcomes: Analysis using individual participant data from randomised trials. BMC Pregnancy Childbirth [Internet]. 2019 [cited 2020 Jan 10]; 19(322). DOI: https://doi.org/10.1186/s12884-019-2472-7.

4. IOM (Institute of Medicine). Weight Gain During Pregnancy: Reexamining the Guidelines. Washington (DC): The National Academies Press [Internet]. 2009 [cited 2019 Nov 15]; DOI: https://doi.org/10.17226/12584.

5. Ministério da Saúde (Br). Atenção ao pré-natal de baixo risco. Brasilia (DF): Editora do Ministério da Saúde; 2012 . 316 p. [cited 2019 Nov 15] Available from: http://bvsms.saude.gov.br/bvs/publicacoes/cadernos_atencao_basica_32_prenatal.pdf.

6. Thompson AM, Thompson JA. An evaluation of whether a gestational weight gain of 5 to $9 \mathrm{~kg}$ for obese women optimizes maternal and neonatal health risks. BMC Pregnancy Childbirth [Internet]. 2019 [cited 2020 Jan 09]; 19(1). DOI: https://doi.org/10.1186/s12884-019-2273-z.

7. Alves P, Malheiro MF, Gomes JC, Ferraz T, Montenegro N. Risks of Maternal Obesity in Pregnancy: A Case-control Study in a Portuguese Obstetrical Population. Rev. Bras. Ginecol. e Obstet. [Internet]. 2019 [cited 2020 Fev 20]; 41(12):682-7. DOI: http://doi.org/10.1055/s-0039-3400455.

8. Ferreira LA de $\mathrm{P}$, Piccinato $\mathrm{C}$ de A, Cordioli E, Zlotnik E. Pregestational body mass index, weight gain during pregnancy and perinatal outcome: a retrospective descriptive study. Einstein [Internet]. 2020 [cited 2020 Jan 15]; 18:1-6. DOI: http://dx.doi.org/10.31744/einstein_journal/2020ao4851.

9. Marques DSM, Siqueira HGR, Cruz MA, Vieira MS V, Apolônio RSPT, Esteves APVS. The relationship between pre-eclampsia and obesity: a integration review. Rev Cad Med [Internet]. 2019 [cited 2020 Jan 10]; 2(2):56-62. Available from: http://www.revista.unifeso.edu.br/index.php/cadernosdemedicinaunifeso/article/view/1394.

10. Lima MRG de, Coelho ASF, Salge AKM, Guimarães JV, Costa PS, Sousa TCC de, et al. Maternal changes and puerperal pregnancy outcome in maternal death occurrence. Cad. Saúde Coletiva [Internet]. 2017 [cited 2019 Dec 10]; 25(3):324-31. DOI: https://doi.org/10.1590/1414-462×201700030057.

11. Pires C da C, Rodrigues ML, Capelli J de CS, Santos MMA de S, Baião MR. Nutritional attention and food practices in the perspective of overweight pregnant women. Demetra [Internet]. 2020 [cited 2020 May 06]; 15(e40566). DOI: https://doi.org/10.12957/demetra.2020.40556.

12. Flores-Ortiz R, Malta DC, Velasquez-Melendez G. Adult body weight trends in 27 urban populations of Brazil from 2006 to 2016 : A population-based study. PLoS One [Internet]. 2019 [cited 2019 Dec 10]; 14(3):e0213254. DOI: https://doi.org/10.1371/journal.pone.0213254.

13. Araújo FG, Velasquez-Melendez G, Felisbino-Mendes MS. Prevalence trends of overweight, obesity, diabetes and hypertension among Brazilian women of reproductive age based on sociodemographic characteristics. Health Care Women Int. [Internet]. 2019 [cited 2020 Mai 06]; 40(4):386-406. DOI: https://doi.org/10.1080/07399332.2019.1570516.

14. Leal MC, Silva AAM, Dias MAB, Gama SGN, Rattner D, Moreira ME. Birth in Brazil: national survey into labourand birth. Reproductive Health [Internet]. 2012 [cited 2020 Mai 07]; 9(1):15. DOI: https://doi.org/10.1186/1742-4755-9-15.

15. Barros AJ, Hirakata VN. Alternatives for logistic regression in cross-sectional studies: an empirical comparison of models that directly estimate the prevalence ratio. BMC Med Res Methodol [Internet]. 2003 [cited 2020 May 07]; 3. DOI: https://doi.org/10.1186/1471-2288-3-21.

16. Parker HW, Tovar A, McCurdy K, Vadiveloo M. Associations between pre-pregnancy BMI, gestational weight gain, and prenatal diet quality in a national sample. PLoS One [Internet]. 2019 [cited 2020 May 10]; 14(10). DOI: https://doi.org/10.1186/14712288-3-21.

17. Alves P, Malheiro MF, Gomes JC, Ferraz T, Montenegro N. Risks of Maternal Obesity in Pregnancy: A Case-control Study in a Portuguese Obstetrical Population. Rev. Bras. Ginecol. Obs. [Internet]. 2019 [cited 2020 May 07]; 41(12):682-7. DOI: https://doi.org/10.1055/s-0039-3400455. 
18. Kale PL, Jorge MHP de M, Fonseca SC, Cascão AM, da Silva KS, Reis AC, et al. Deaths of women hospitalized for childbirth and abortion, and of their concept, in maternity wards of Brazilian public hospitals. Cienc e Saude Coletiva [Internet]. 2018 [cited 2020 May 10]; 23(5):1577-90. DOI: https://doi.org/10.1590/1413-81232018235.18162016.

19. Ferreira RAB, Benicio MHD. Obesity in Brazilian women: association with parity and socioeconomic status. Rev. Panam. Salud Publica [Internet]. 2015 [cited 2020 Fev 21]; 37(4/5). Avaliable from: https://www.scielosp.org/article/rpsp/2015.v37n4-5/337$342 /$.

20. Rebesco DB, Souza WC, Lima VA, Grzelczak MT, Frasson AC, Mascarenhas LPG. Physical exercise in pregnancy action: a review of study. Arch Heal Investig [Internet]. 2016 [cited 2020 Mai 10]; 6(6):311-7. DOI: http://dx.doi.org/10.21270/archi.v5i6.1707.

21. Gonçalves DF, Teixeira MTB, Silva GA, Duque KCD, Machado MLSM, Ribeiro LC. Reproductive factors associated with overweight in adult women attended by the Family Health Strategy. Ciênc. Saúde Coletiva [Internet]. 2020 [cited 2020 Apr 03]; 25(8):3009-16. DOI: http://dx.doi.org/10.1590/1413-81232020258.30642018.

22. Silva ABJ, Oliveira AVK, Silva JD, Quintaes KD, Fonseca VAS, Nemer ASA. Relationship between alcohol consumption by female college students and body adiposity. J. bras. psiquiatr [Internet]. 2011 [cited 2020 Apr 10]; 60(3): 210-215. DOI: https://doi.org/10.1590/S0047-20852011000300010.

23. Meucci RD, Saavedra JS, da Silva ES, Branco MA, de Freitas JN, dos Santos M, et al. Alcohol intake during pregnancy among parturients in southern Brazil. Rev. Bras. Saude Matern. Infant. [Internet]. 2017 [cited 2020 Apr 10]; 17(4):653-61. DOI: http://dx.doi.org/10.1590/1806-93042017000400003.

24. Gomes CBA, Dias RS, Silva WGB, Pacheco MAB, Sousa FGM, Loyola CMD. Prenatal nursing consultation: narratives of pregnant women and nurses. Texto contexto - enferm. [Internet] 2019 [cited 2020 May 15]; 28:e20170544. DOI: https://doi.org/10.1590/1980-265x-tce-2017-0544.

25. Leal M do C, Szwarcwald CL, Almeida PVB, Aquino EML, Barreto ML, Barros F, et al. Reproductive, maternal, neonatal and child health in the 30 years since the creation of the Unified Health System (SUS). Cien Saude Colet. [Internet] 2018 [cited 2020 May 15]; 23(6):1915-28. DOI: https://doi.org/10.1590/1413-81232018236.03942018.

26. Souza VB, Silva JS, Barros MC, Freitas PSP. Soft technologies in health to potentize the quality of care to pregnant women. Rev. enferm. UFPE Online [Internet]. 2014 [cited 2020 Apr 15]; 8(5):1388. DOI: https://doi.org/10.5205/reuol.5863-50531-1ED.0805201437.

27. Alves ACP, Figueiredo M de FER, Sousa NPL de, Oliveira CJ de, Oliveira DR de, Sousa WM de. Application of prenatal care light technology: focus on pregnant women's perception. Rev. enferm. UERJ [Internet]. 2013 [cited 2020 Apr 15]; 21(1):648-53. Avaliable from: https://www.e-publicacoes.uerj.br/index.php/enfermagemuerj/article/view/10043/8071.

28. Errico L de SP de, Bicalho PG, Oliveira TCFL de, Martins EF. The work of nurses in high-risk prenatal care from the perspective of basic human needs. Rev. Bras. Enferm. [Internet] 2018 [cited 2020 May 10]; 71:1257-64. DOI: http://dx.doi.org/10.1590/00347167-2017-0328.

29. Liu L, Coenen A, Tao H, Jansen K, Jiang A, Jiang A. Developing a prenatal nursing care International Classification for Nursing Practice catalogue. Int. Nur. Rev. [Internet] 2016 [cited 2020 May 13]; 1-8. DOI: http://doi.org/10.1111/inr.12325.

30. de Oliveira RR, Melo EC, Novaes ES, Ferracioli PLRV, Mathias TA de F. Factors associated to Caesarean delivery in public and private health care systems. Rev Esc Enferm USP [Internet]. 2016 [cited 2020 May 13]; 50(5):734-41. DOI: https://dx.doi.org/10.1590/s0080-623420160000600004.

31. Brenes-Monge A, Saavedra-Avendaño B, Alcalde-Rabanal J, Darney BG. Are overweight and obesity associated with increased risk of cesarean delivery in Mexico? A cross-sectional study from the National Survey of Health and Nutrition. BMC Pregnancy Childbirth [Internet]. 2019 [cited 2020 May 21]; 19(1). DOI: https://doi.org/10.1186/s12884-019-2393-5. 